\title{
The Cardiovascular and Perceptual Response to Very Low Load Blood Flow Restricted Exercise
}

\author{
Authors \\ Matthew B Jessee, Scott J Dankel, Samuel L Buckner, J. Grant Mouser, Kevin T Mattocks, Jeremy P Loenneke
}

\begin{abstract}
Affiliations
Kevser Ermin Applied Physiology Laboratory, University of Mississippi, Health, Exercise Science, and Recreation

Management, University, United States

Key words

occlusion training, vascular occlusion, ischemic exercise, arterial occlusion
\end{abstract}

accepted after revision 12.04.2017

\author{
Bibliography \\ DOI https://doi.org/10.1055/s-0043-109555 \\ Published online: 26.6.2017 \\ Int J Sports Med 2017; 38: 597-603 \\ (c) Georg Thieme Verlag KG Stuttgart · New York \\ ISSN 0172-4622 \\ Correspondence \\ Dr. Jeremy Paul Loenneke \\ University of Mississippi \\ Health, Exercise Science, and Recreation Management \\ 231 Turner Center \\ 38677, Oxford \\ United States \\ Tel.: + 1/662/915 5567, Fax: + 1/662/915 5525 \\ jploenne@olemiss.edu
}

\begin{abstract}
This study sought to compare cardiovascular and perceptual responses to blood flow restriction (BFR) exercise using various pressure and load combinations. Fourteen participants completed four sets of BFR elbow flexion using 10, 15 and 20\% 1RM with 40 and $80 \%$ arterial occlusion pressure (AOP). AOP was measured before and after exercise. Perceived exertion (RPE) and discomfort were assessed before exercise and after each set. Data presented as mean $(95 \% \mathrm{Cl})$, except for RPE and discomfort: $25^{\text {th }}, 50^{\text {th }}, 75^{\text {th }}$ percentiles. AOP increased post-exercise $(p<0.001)$ with larger magnitudes seen when increasing load and pressure $(p<0.001)$ [e. g., 10/40 $\Delta$ AOP: $21(10,32)$ mmHg vs. $20 / 80 \triangle$ AOP: $62(45,78) \mathrm{mmHg}$, which also augmented RPE $(p<0.001)$ [e. g., 4th set $10 / 40:(7,8.5,12)$ vs. 4th set 20/80: $(12.75,15.5,17.25)]$ and discomfort $(p<0.001)$ [e. $g ., 4^{\text {th }}$ set $10 / 40:(0.75,2,4.25)$ vs. $4^{\text {th }}$ set $20 / 80:(4.25,6$, 8$,$) ]. Volume increased via greater loads (\mathrm{p}<0.001)$, and participants only reached failure during $20 \% 1 \mathrm{RM}$ conditions [20/40: $74(74,75)$ repetitions; 20/80: $71(68,75)$ repetitions]. When performing BFR exercise with very low loads the magnitudes of the cardiovascular and perceptual responses are augmented by increasing the load and by applying a higher relative pressure.
\end{abstract}

\section{Introduction}

Blood flow restriction in combination with low load resistance training has been shown to increase muscle size comparably to high load resistance training [15]. As high load resistance exercise may place a large mechanical stress on the joints, blood flow restriction exercise may be an efficacious alternative for a variety of populations (e. g., elderly, injured, athletic) aiming to increase or maintain muscular fitness. However, for a training modality to be effective it must be adhered to by the target population. Thus, it must be safe and should be perceived favorably by those participating in the particular modality of training. Recently, concerns about an exaggerated cardiovascular response following blood flow restriction exercise have been proposed [24] as an argument against the widespread implementation of this training method. In an effort to resolve these concerns, it has been recommended that restriction pressures be made relative to the cuff being used $[11,18]$ and the limb circumference $[11,17]$ of each individual. This not only leads to the stimulus being relative to individual differences, but it also ensures practitioners are not inadvertently applying an occlusive stimulus [12].

Thus far, the cardiovascular response to blood flow restriction exercise in the upper body using these relative applied pressures has only been characterized when exercising with $30 \%$ of one repetition maximum (1RM) [4] even though loads lower than that could be effective for increasing muscle size $[1-3,13,14]$. Some evidence suggests that higher pressures are needed to elicit muscle growth when using very low loads (20\% 1RM) [16]. It remains to be seen, however, how moderate and higher relative pressures augment the cardiovascular response when exercising at such a low load. Additionally, the perceptual responses to varying levels 
of relative pressure with these very low loads may dictate compliance as participants may be less apt to engage in a particular form of exercise that they perceive as intolerable. The aim of this study was to determine the cardiovascular and perceptual responses to blood flow restriction exercise using very low loads in conjunction with moderate and high relative restrictive pressures.

\section{Methods}

\section{Participants}

Fourteen participants ( 11 males and 3 females) volunteered to participate in the study. All had performed resistance training at least twice a week for the previous six months and were familiar with the dumbbell elbow flexion (biceps curl) exercise. Participants were eligible for inclusion to the study if they: were within the age range of $18-35$ years, did not use tobacco, had a $\mathrm{BMI}<30$, abstained from exercise in the $24 \mathrm{~h}$ previous to testing, abstained from caffeine in the $8 \mathrm{~h}$ prior to testing, ingested no food within $2 \mathrm{~h}$ of testing, avoided the consumption of alcohol in the previous $24 \mathrm{~h}$, and if they were free of any orthopedic injury preventing exercise. Additionally, participants had to meet less than two of the following risk factors for thromboembolism to be considered for inclusion: currently taking birth control, diagnosed with Crohn's disease, past fracture of hip, pelvis, or femur, major surgery within the last 6 months, varicose veins, family or personal history of deep vein thrombosis, or family or personal history of pulmonary embolism [22]. All participants were informed of the experimental procedures as well as any potential risks that were associated with the study before giving written informed consent. The University's Institutional Review Board approved this study, and it meets the ethical standards set forth by the International Journal of Sports Medicine [10].

\section{Experimental design}

Participants made four visits to the laboratory, each one at the same time of day, separated by at least five but no more than ten days. Visit 1 consisted of paperwork followed by measurements of height, body mass, standing arterial occlusion pressure, and elbow flexion (biceps curl) one repetition maximum (1RM). Participants were then familiarized with blood flow restriction exercise. For each visit thereafter, two of the six possible conditions were tested (one with each arm) until all six conditions were applied over the three different visits. Each condition was a different combination of load $(10,15$, and $20 \%$ of $1 R M)$ and relative restriction pressure (40 and $80 \%$ AOP). For each testing condition, the participant began by resting quietly (10 min before condition 1 , and 15 min before condition 2) followed by a measurement of standing arterial occlusion pressure on each arm using a hand held Doppler probe. Afterwards, participants performed four sets (goal repetitions: $30,15,15,15$ ) of unilateral elbow flexion exercise in combination with blood flow restriction. During the $30 \mathrm{~s}$ of rest observed between each set of exercise, participants were asked for their ratings of perceived exertion (RPE) and level of discomfort. Immediately following the last set of exercises, standing arterial occlusion pressure was measured again in the exercised arm. Participants then rested quietly for 15 min after which all testing was repeated in the opposite arm only, using a new combination of load and pressure. Arterial occlu- sion pressure was reassessed (in the arm to be exercised) before beginning condition 2 . This was to ensure that the cardiovascular response was not still elevated due to having undergone condition 1. After both conditions were tested (one per arm), the visit was over and the next four conditions were completed during the subsequent visits (two conditions per visit). The order of the arms, loads, and restriction pressures used for visits 2-4 were randomized during visit 1 using a random sequence generator. All restrictive pressures applied were based upon the arterial occlusion pressure measurement taken immediately prior to each exercising condition.

\section{Arterial occlusion pressure}

Standing arterial occlusion pressure was measured before each bout of exercise, and immediately after exercise. A $5 \mathrm{~cm}$ wide, nylon cuff (SC5 Hokanson, Bellevue, WA) was placed on the most proximal portion of the participant's upper arm. A hand-held Doppler probe (MD6, Hokanson, Bellevue, WA) was placed at the wrist over the radial artery until an auditory signal of blood flow was found. The cuff was slowly inflated using an E20 Rapid Cuff Inflator (Hokanson, Bellevue, WA) until there was no longer an indication of blood flow from the Doppler probe. The lowest cuff inflation pressure at which the blood flow distal to the cuff was no longer detectable was defined as arterial occlusion pressure. The post-exercise arterial occlusion measurement was taken immediately after exercise by increasing the inflation pressure off the cuff, which was already inflated during the bout of exercise. Once arterial occlusion was determined the cuff was immediately deflated and removed from the upper arm.

\section{One-repetition maximum}

One-repetition maximum for unilateral elbow flexion was assessed in both arms on visit 1 , in order to determine the appropriate relative load for all conditions. Participants began the test following a warm up of 5-10 repetitions using $30 \%$ of an assumed maximum. Each attempt during testing began with participants standing with their feet shoulder width apart, their heels and back against a wall, and the arm fully extended and supinated by their side. Once in proper position they were handed a loaded dumbbell and encouraged to complete a full range of elbow flexion while maintaining an upright position. Attempts began by completing one repetition at an estimated $60-75 \%$ of maximum, and the load was progressively increased until the participant was unable to lift a load greater than their previous successful attempt. After each attempt participants were given a rest period before attempting the next load. An attempt was deemed unsuccessful, if the participant could not complete the full range of motion, or if they were unable to maintain strict form with their heels and back against a wall. One-repetition maximum was determined as the greatest load a participant was able to lift properly through a full range of motion.

\section{Blood flow restriction protocol}

Blood flow restriction was applied by placing a $5 \mathrm{~cm}$ wide nylon cuff (SC5, Hokanson, Bellevue, WA) to the proximal portion of the exercising limb and inflating it to either 40 or $80 \%$ of pre-exercise arterial occlusion. These particular pressures were investigated as they are often used in the blood flow restriction research 

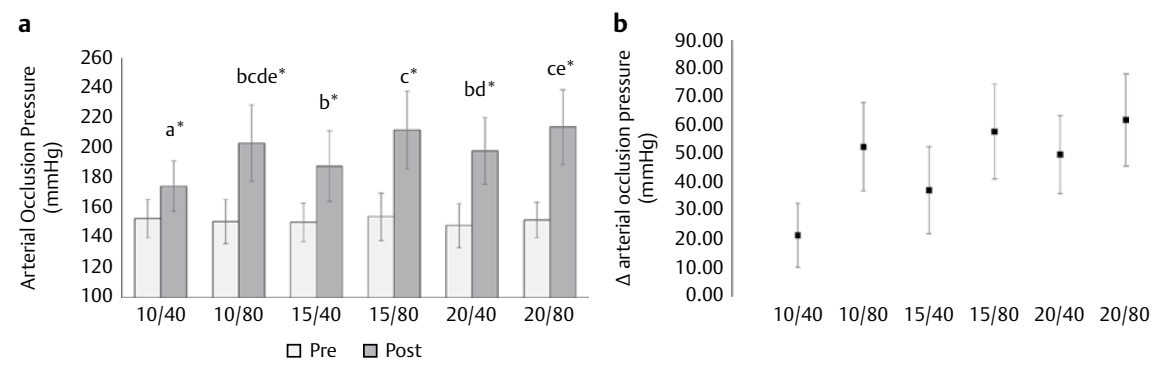

Fig. 1 Arterial occlusion pressure $\mathbf{a}$ and the change $\mathbf{b}$ before

(pre) and immediately following (post) blood flow restricted exercise. Conditions are labeled as percentages of one-repetition maximum/percentage of arterial occlusion pressure. Different letters indicate significant differences across conditions for the post time point, and * indicates significant differences between pre and post measurements $(p \leq 0.05)$. If at least one letter is the same, those conditions are not significantly different. Data presented as mean $(95 \% \mathrm{Cl})$.

$[4,7,14,27]$ and both have been shown to elicit muscle adaptation $[8,15]$. After inflation the participants performed four sets of elbow flexion with one of three experimental loads $(10,15$, or $30 \%$ of $1 \mathrm{RM})$. The goal repetitions were modeled from a commonly used blood flow restriction protocol of $30,15,15,15$ with 30 s of rest between each set [9]. Each repetition was performed to a metronome cadence of $1 \mathrm{~s}$ for the concentric portion and $1 \mathrm{~s}$ for the eccentric portion of the lift.

\section{Ratings of Perceived Exertion (RPE) and discomfort}

Participants were informed in depth on how to rate their exertion (RPE) and discomfort to ensure they understood the scale being used. In short, participants were told that a rating of 6 meant they were not exerting themselves at all, and a rating of 20 meant that they were giving maximal effort and would be unable to exert themselves any further. Individuals were asked to rate their level of exertion using the standard Borg 6-20 scale [5, 20]. This was asked immediately prior to beginning exercise, and then again immediately following each set of exercise. A rating of discomfort was obtained using Borg's Discomfort Scale $($ CR10 + ) [5, 20]. It was explained to participants that the scale was rated from $0-10$ with a score of 10 representing their previously worst felt discomfort. They were then instructed that a rating of 10 was their reference point, and they could exceed 10 , if the discomfort they felt was greater than what they have ever felt before. Participants were then asked whether they had any questions. To ensure that all participants fully understood the scale prior to exercise, they were provided the opportunity to request any clarification and they were asked if they understood how to rate their level of discomfort. Ratings of discomfort were taken immediately before exercise, as well as $20 \mathrm{~s}$ after sets 1,2 and 3, and immediately after set 4 . Discomfort was taken $20 \mathrm{~s}$ after each set because participants in previous studies anecdotally noted greater discomfort later in the rest periods and it was thought that this provided a more accurate representation of the level of discomfort caused by the exercise protocol [8].

\section{Statistical analyses}

All data were analyzed using SPSS 23.0 statistical software package (IBM, Chicago, II, USA). To detect any interaction effect of con- dition and time for arterial occlusion, a 6 (condition) $\times 2$ (time) repeated measures analysis of variance (ANOVA) was used. If there was a significant interaction effect, a one-way repeated measures ANOVA was used to reveal differences across conditions within each time point, and a paired samples t-test was used to find differences between time points within each condition. For comparisons of perceptual responses (RPE and discomfort), a Friedman non-parametric test was used to reveal any differences between conditions within each time point (pre, set 1 , set 2 , set 3, set 4). If a significant difference was revealed, Wilcoxon related samples non-parametric tests were used to find where those differences occurred. To compare exercise volumes across conditions, a one-way repeated measures ANOVA was used. All data are presented as means and $95 \%$ confidence intervals. For perceptual responses data are represented as $25^{\text {th }}, 50^{\text {th }}$, and $75^{\text {th }}$ percentiles. Statistical significance was set a priori at an alpha level of 0.05 .

\section{Results}

\section{Participant characteristics}

In total, 3 females and 11 males [mean (95\% Cl); Age: $24(22,26)$ years; height: $175.2(169.0,181.3) \mathrm{cm}$; body mass: 83.6 (75.3, 92.0) kg; 1-RM left arm 25.4 (20.6, 30.2) kg; 1-RM right arm 26.1 $(21.0,32.2) \mathrm{kg}]$ completed all testing conditions.

\section{Arterial occlusion pressure}

For arterial occlusion pressure, there was a significant interaction effect of condition $x$ time $(p<0.001)$. Follow-up tests revealed that arterial occlusion pressure was increased from pre to post $(p<0.001)$ across all conditions. There were no significant differences across condition at pre $(p=0.659)$. At post, however, there were significant differences ( $\vee$ Fig. $\mathbf{1 a}, \mathrm{p}<0.001$ ) with the arterial occlusion being augmented by the greater applied pressure $(80>40 \%)$ and in some cases a heavier exercise load $(20 \%$ $1 \mathrm{RM}>15 \% 1 \mathrm{RM}>10 \% 1 \mathrm{RM})$. The mean change $(95 \% \mathrm{Cl})$ in arterial occlusion pressure from pre to post for each condition can be found in > Fig. 1b. Although applied pressure remained the same, it was based upon the percentage of pre-arterial occlusion, and since ar- 
terial occlusion increased post exercise the percentage of pressure being applied was lower ( $\triangleright$ Fig. 2 ). It should be noted that for the post measurement of arterial occlusion pressure, one participant did not reach full occlusion following exercise in the 10/80 condition. This is due to the limitation of the E20 Rapid Cuff Inflator, which does not exceed $300 \mathrm{mmHg}$. Thus, for that measure we recorded arterial occlusion as $300 \mathrm{mmHg}$, and it was used in the analysis.

\section{Ratings of Perceived Exertion (RPE)}

There were no significant differences in RPE across conditions at pre $(\triangleright$ Table 1, $p=0.306)$. Significant differences were revealed across

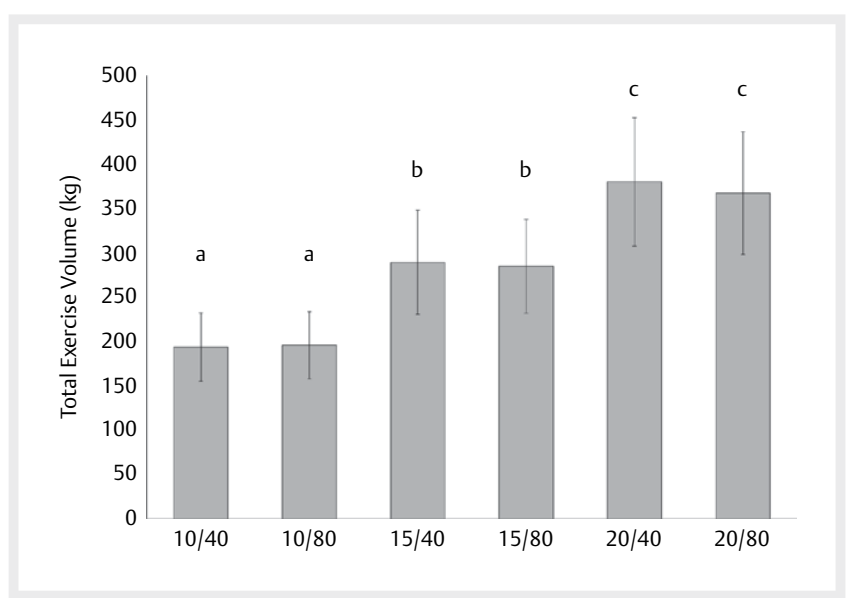

- Fig. 2 Total exercise volume completed across conditions. Conditions are labeled as percentages of one-repetition maximum/percentage of arterial occlusion pressure. Different letters indicate significant differences across conditions $(p \leq 0.05)$. If at least one letter is the same, those conditions are not significantly different. Data presented as mean $(95 \% \mathrm{Cl})$. condition within each set of exercise ( $\triangleright$ Table $1, p<0.001)$ with the RPE generally being higher with greater loads and pressures.

\section{Ratings of discomfort}

Similar to RPE there were no differences in discomfort across conditions at pre ( $\triangleright$ Table $1, p=0.203$ ). There were significant differences across conditions within each set of exercise ( $\triangleright$ Table 1 , $p<0.001$ ) with discomfort generally being higher with greater loads and pressures.

\section{Exercise volume}

For exercise volume there was a significant difference across conditions ( $p<0.001)$. In general, volume was greater with increased load, but it was not attenuated with higher pressures ( $>$ Fig. $\mathbf{3}$ ). Since the changes in total volume are driven by the experimental differences in load, we also chose to compare the total repetitions completed between conditions to determine whether participants were reaching failure in any of the conditions. For total repetitions, there was no significant difference ( $p \geq 0.052)$ across conditions ( $\triangleright$ Table 2), though there were some individuals who were unable to complete the full protocol at 20\% 1RM. For example, one person was unable to complete all repetitions using $20 \% 1$ RM and $40 \%$ BFR and four participants were unable to complete all repetitions using $20 \% 1 \mathrm{RM}$ and $80 \% \mathrm{BFR}$.

\section{Discussion}

In the current study, arterial occlusion pressure significantly increased immediately following blood flow restricted exercise, resulting in the applied pressure dropping to a lower percentage of arterial occlusion. Larger increases in post exercise arterial occlusion pressure were observed with increases in load and applied pressure. Similarly, ratings of perceived exertion and discomfort were greater with increased loads and higher pressure. Taken to-

- Table 1 Perceptual responses to various load and pressure combinations.

\begin{tabular}{|c|c|c|c|c|c|}
\hline \multicolumn{6}{|c|}{ Rating of Perceived Exertion } \\
\hline Load/Pressure & Pre & Set 1 & Set 2 & Set 3 & Set 4 \\
\hline $10 / 40$ & $6,6,6$ & $7,7,8^{\text {a }}$ & $7,7.5,9^{a}$ & $7,8,9.25^{\mathrm{a}}$ & $7,8.5,12^{\text {a }}$ \\
\hline $10 / 80$ & $6,6,6$ & $7,8,9.25$ ab & $8.75,10,11.25^{b}$ & $9.75,11,12.25^{b}$ & $11,11.5,13^{b}$ \\
\hline $15 / 40$ & $6,6,6$ & $7,8,11^{b c}$ & $7.75,10.5,12^{\text {bd }}$ & $8,10.5,13.25^{b}$ & $8.75,11,14.25^{b}$ \\
\hline $15 / 80$ & $6,6,6$ & $8,9.5,12^{c}$ & $10,12,13.25^{c}$ & $11.5,13,14.5^{c}$ & $11,14,16.25^{c}$ \\
\hline $20 / 40$ & $6,6,6$ & $8,9.5,11.25^{c}$ & $10,11,12.5^{\mathrm{cd}}$ & $11,12.5,14.25^{c}$ & $11,13,16^{c}$ \\
\hline $20 / 80$ & $6,6,6$ & $8,11.5,13^{c}$ & $10.5,13,15^{c}$ & $11,14.5,16.25^{c}$ & $12.75,15.5,17.25^{c}$ \\
\hline \multicolumn{6}{|c|}{ Ratings of Discomfort } \\
\hline Load/Pressure & Pre & Set 1 & Set 2 & Set 3 & Set 4 \\
\hline $10 / 40$ & $0,0,0$ & $0,1,2^{\mathrm{a}}$ & $0,1,3^{\text {a }}$ & $0,1,3^{\text {a }}$ & $0.75,2,4.25^{a}$ \\
\hline $10 / 80$ & $0,0,0$ & $1.75,3,3.25^{b}$ & $2.75,3.5,5^{b}$ & $3,5,5^{b}$ & $3.75,5,6.25^{b}$ \\
\hline $15 / 40$ & $0,0,0$ & $1,1,2$ ac & $1,2,3$ ac & $1,2,4.62^{c}$ & $1,2.5,5^{a}$ \\
\hline $15 / 80$ & $0,0,0$ & $1.75,4,5^{b}$ & $4,5.5,6.25^{d}$ & $5.5,6,7^{d}$ & $5.5,6.5,7.25^{c}$ \\
\hline $20 / 40$ & $0,0,0$ & $1,2,3^{c}$ & $1,3,4$ bc & $1,2.5,6^{c}$ & $1.75,3.5,6.25^{d}$ \\
\hline $20 / 80$ & $0,0,0$ & $2.5,3.5,5^{b}$ & $3.5,5.5,7^{d}$ & $3.75,5.5,7.25^{d}$ & $4.75,6,8^{c}$ \\
\hline
\end{tabular}


gether the data suggests that both the cardiovascular and perceptual responses to blood flow restriction exercise with very low loads are augmented by increasing the load lifted and increasing the relative restriction pressure applied.

\section{Cardiovascular response}

In order to determine the cardiovascular response to blood flow restricted exercise using very low loads, we measured the post arterial occlusion pressure immediately following the cessation of the last set of exercises. Although this is not necessarily a traditional measure of systolic blood pressure since it is measured with a nonstandard narrow cuff $(5 \mathrm{~cm})$ [17], it is similar, and allows us to capture the cardiovascular response to this type of exercise (i. e. unilateral bicep curl) without deflating and removing the cuff potentially missing the true cardiovascular response. The pressure required to completely occlude arterial blood flow increased following exercise in all conditions and was augmented by increasing the load and by applying a higher pressure. The mean change in arterial occlusion pressure ranged from $21 \mathrm{mmHg}$ in the $10 / 40$ condition to $62 \mathrm{mmHg}$ in the $20 / 80$ condition. The increase at lower loads and pressures is similar to the average increase of 24 or $26 \mathrm{mmHg}$ (depending on the cuff width) observed immediately following (prior to deflation) bilateral leg extension exercise with $20 \%$ 1 RM [23]. It should be noted that the pressure for the Rossow et al. study was based on brachial systolic blood pressure, which makes it difficult to determine the relative pressure applied to the lower body $[17,18]$.

In the upper body, Brandner et al. [6] found that systolic blood pressure changes following traditional high load unilateral bicep curl exercise ( $80 \%$ 1RM) were similar (approx. $50 \mathrm{mmHg}$ change) to a continuous blood flow restriction protocol using $20 \% 1 \mathrm{RM}$. When comparing this cardiovascular response to blood flow restricted exercise using a relative pressure of $40 \%$ arterial occlusion, Barnett et al. [4] observed a mean increase in arterial occlusion pressure of approx. $31 \mathrm{mmHg}$ immediately following unilateral bicep curl exercise. That increase resulted in the applied pressure of $40 \%$ arterial occlusion before exercise to decrease to approx. $32 \%$ of arterial occlusion post exercise, which was similar to the moderate pressure conditions in the current study, whereas the high pressure conditions ( $80 \%$ of AOP) decreased to around $56-60 \%$ of arterial occlusion. This may be important given the possible existence of a minimum pressure threshold in which pressure is required to be beneficial for adaptation [21]. If this threshold does in fact exist, it may be necessary to use a higher restriction pressure to keep the applied pressure above that threshold. Although higher pressures may not be necessary with loads such as $30 \%$ of 1 RM or greater [8], they might be necessary with loads less than $30 \% 1$ RM. For example, a long term training study found that muscle size increases were lower when using $20 \%$ of 1 RM in combination with a moderate blood flow restriction pressure ( $40 \%$ arterial occlusion) compared to a group using the same load combined with a high pressure ( $80 \%$ arterial occlusion) [16]. Even though applying a higher pressure combined with very low loads does augment the cardiovascular response (mean increase of $21-62 \mathrm{mmHg}$ ), it should be noted that the magnitude of change is similar to that observed with traditional high load resistance training (approx. $50 \mathrm{~mm} \mathrm{Hg}$ ) [6]. Thus, resistance exercise using very low loads in

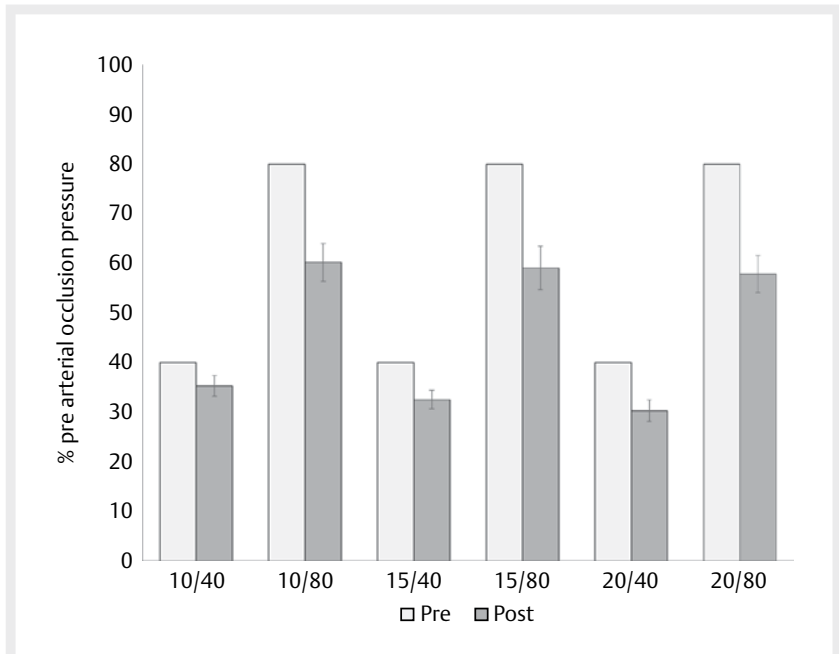

- Fig. 3 Applied pressure relative to arterial occlusion pressure before (pre) and immediately following (post) blood flow restricted exercise. Conditions are labeled as percentages of one-repetition maximum/percentage of arterial occlusion pressure. Data presented as mean $(95 \% \mathrm{Cl})$.

- Table 2 Total repetitions completed.

\begin{tabular}{|l|c|c|c|c|}
\hline $\begin{array}{l}\text { Condi- } \\
\text { tion }\end{array}$ & Mean & Cl & $\begin{array}{l}\text { Mini- } \\
\text { mum }\end{array}$ & $\begin{array}{c}\text { Maxi- } \\
\text { mum }\end{array}$ \\
\hline $10 / 40$ & 75 & $(75,75)$ & 75 & 75 \\
\hline $10 / 80$ & 75 & $(75,75)$ & 75 & 75 \\
\hline $15 / 40$ & 75 & $(75,75)$ & 75 & 75 \\
\hline $15 / 80$ & 75 & $(75,75)$ & 75 & 75 \\
\hline $20 / 40$ & 74 & $(74,75)$ & 72 & 75 \\
\hline $20 / 80$ & 71 & $(68,75)$ & 58 & 75 \\
\hline
\end{tabular}

Conditions are labeled as percentages of one-repetition maximum/ percentage of arterial occlusion pressure

combination with moderate or high relative BFR pressures does not seem likely to pose an increased cardiovascular health risk when compared to traditional resistance exercise.

\section{Perceptual response}

To make inferences about possible adherence to a blood flow restriction training protocol, participants were asked to rate their level of perceived exertion and discomfort following each exercise condition, using a combination of very low loads with moderate or high restriction pressures. Ratings of perceived exertion were greater with increases in load and pressure, with the exception of $20 \%$ 1 RM conditions where RPE was similar between moderate and high pressure conditions for set 4 . Although RPE is expected to be greater when increasing the load, it was augmented by pressure as well. Blood flow restriction may exacerbate RPE over traditional, loadmatched exercise by stimulating cutaneous afferent nerves. In turn, this may lead to an increased central descending drive causing an exaggerated perception of work [26]. When compared to a control group using the same load (20\% 1RM), Yasuda et al. [29] observed an increased RPE when applying blood flow restriction, but there were no differences when increasing the pressure from $98 \mathrm{mmHg}$ 
to $121 \mathrm{mmHg}$, or $147 \mathrm{mmHg}$, which is similar to our observations for the $20 \% 1$ RM conditions. Although applying blood flow restriction does increase RPE over a load matched control condition, it is still significantly lower compared to a traditional high load exercise protocol to volitional failure [28]. When comparing across moderate and high relative pressures, Loenneke et al. [20] observed no differences in RPE due to pressure. The same trend was observed in the current $20 \% 1 \mathrm{RM}$ conditions, whereas in the $10 \% 1 \mathrm{RM}$ and $15 \% 1$ RM conditions RPE was augmented by a higher pressure. Thus, the perceived exertion to exercise with very low loads (less than $20 \% 1$ RM) may be augmented by increasing pressure.

Ratings of discomfort were increased in each relative loading condition when applying a high pressure versus a moderate pressure. Higher pressures may result in a greater accumulation of metabolic byproducts within the limb [28], increasing the perception of discomfort through a stimulation of group III and IV afferent fibers [25]. However, previous studies have not always found a relationship between whole blood lactate and ratings of discomfort [19], suggesting that there may be other underlying physiological and/or psychological mechanisms associated with discomfort during blood flow restricted exercise. When comparing a range of moderate to high relative pressures during blood flow restriction exercise with $30 \% 1$ RM, Loenneke et al. [20] found no real differences in discomfort. This is in contrast to our observations and those of Counts et al. [8], where discomfort was greater in a high pressure condition versus a moderate pressure condition. Discrepancies in studies may be due to baseline strength of the participants or the timing of when participants were asked their level of discomfort. Relative to the Counts investigation [8], participants in the Loenneke study [20] were lifting a higher absolute load, and it is possible that at $30 \% 1 \mathrm{RM}$ this may, in itself, contribute to greater discomfort and mask any effect that pressure may have. In participants with high baseline strength, such as those in the present investigation, augmented discomfort by increasing pressure may only be observed when using loads less than $30 \% 1 \mathrm{RM}$. Another possibility is that Loenneke et al. [20] asked participants to rate their discomfort immediately following each set of exercise, whereas the current participants were asked twenty seconds after the cessation of each set. This time point may better capture the true discomfort of restricting blood flow as the skeletal muscle pump is inactive and very little blood, if any at all, is leaving the exercised limb. Altogether RPE and discomfort are greater when increasing load and pressure.

\section{Repetitions and volume}

Previous studies have shown that total exercise volume using $30 \%$ 1 RM was attenuated by increasing the percentage of relative blood flow restriction pressure applied [8, 20]. In the current study, exercise volume was not attenuated by pressure for the majority of participants. Given the fact that participants were lifting such low loads and were able to complete nearly all repetitions for each condition the differences in volume are explained by the increase in load. For that reason, we also chose to investigate the total number of repetitions completed and found that not everyone was able to complete all of the repetitions at the $20 \%$ load. Thus, if implementing a common goal repetition scheme of 30-15-15-15 with blood flow restriction exercise at 20\% 1RM, applying a higher pres- sure may induce fatigue more quickly than moderate pressures, which may be beneficial for certain populations looking to increase or maintain muscle fitness with minimal joint stress.

\section{Limitations}

The current study is not without limitations. First, there was no quantification of blood flow, only the detection of blood flow cessation with a handheld Doppler probe, which means $40 \%$ or $80 \%$ of arterial occlusion pressure is not necessarily a $40 \%$ or $80 \%$ reduction in blood flow. Second, the cuff used to determine the cardiovascular response was a narrow $5 \mathrm{~cm}$ cuff, and responses could be different with varying cuff widths, including that of a clinical blood pressure cuff. However, we believe that the best way to capture the cardiovascular response immediately after exercise was to use the cuff already applied and inflated for blood flow restriction and examine the arterial occlusion pressure changes. Finally, the testing of two conditions in the same day can be viewed as a possible limitation. The reperfusion of blood flow and circulating endothelial factors as a result from the first exercise bout could have influenced the second condition, which was tested in the contralateral arm. However, rest was taken between the conditions to allow for a washout period. Since AOP was assessed before all exercise conditions, and immediately before the second exercise protocol, we ran a retrospective dependent t-test to determine that there were no statistically significant differences between AOP of arm 2 assessed before any exercise and after the rest period following condition 1. The mean differences $(95 \% \mathrm{Cl})$ between AOP measurements were $2.7(-1.8,7.4) \mathrm{mmHg}$ for visit $1(p=0.219), 1.7(-8.8$, $5.4) \mathrm{mmHg}$ for visit $2(p=0.614)$, and $1.1(-6.7,4.4) \mathrm{mmHg}$ for visit $3(p=0.666)$. Therefore, if in fact there was a residual effect of condition 1 on condition 2 , it was minimal in comparison to what was observed from the exercise protocol itself.

\section{Conclusions}

The results of the current study have shown that when resistance exercise is performed using very low loads in combination with blood flow restriction the cardiovascular and perceptual responses are augmented by an increase in the relative load being used for exercise, as well as an increase in the relative pressure being applied for blood flow restriction. When compared with previous studies implementing traditional high load training, the magnitude of the cardiovascular response to blood flow restriction is similar and does not appear to pose an additional safety concern. Although RPE is augmented by load and pressure, it is less than that previously observed with high load training, suggesting that RPE may not be a limiting factor of participant adherence; it could, however, be limited by discomfort which was also augmented with higher pressures. Blood flow restriction training studies using very low loads with moderate and high pressures should be performed to determine the long term effects on muscle adaptation, as well as cardiovascular and perceptual responses.

\section{Acknowledgements}

The authors do not have any affiliations, grants, or funding to acknowledge. 


\section{References}

[1] Abe T, Fujita S, Nakajima T, Sakamaki M, Ozaki H, Ogasawara R, Sugaya M, Kudo M, Kurano M, Yasuda T, Sato Y, Ohshima H, Mukai C, Ishii N. Effects of low-intensity cycle training with restricted leg blood flow on thigh muscle volume and vo2max in young men. J Sports Sci Med 2010; 9: 452-458

[2] Abe T, Kearns CF, Sato Y. Muscle size and strength are increased following walk training with restricted venous blood flow from the leg muscle, Kaatsu-walk training. J Appl Physiol 2006; 100: 1460-1466

[3] Abe T, Sakamaki M, Fujita S, Ozaki H, Sugaya M, Sato Y, Nakajima T. Effects of low-intensity walk training with restricted leg blood flow on muscle strength and aerobic capacity in older adults. J Geriatr Phys Ther 2010; 33: 34-40

[4] Barnett BE, Dankel S], Counts BR, Nooe AL, Abe T, Loenneke JP. Blood flow occlusion pressure at rest and immediately after a bout of low load exercise. Clin Physiol Funct Imaging 2016; 36: 436-440

[5] Borg G. Borg's Perceived exertion and pain scales. Champaign, IL: Human Kinetics; 1998

[6] Brandner CR, Kidgell D], Warmington SA. Unilateral bicep curl hemodynamics: Low-pressure continuous vs. high-pressure intermittent blood flow restriction. Scand J Med Sci Sports 2015; 25: 770-777

[7] Buckner SL, Dankel S], Counts BR, Jessee MB, Mouser JG, Mattocks KT, Laurentino GC, Abe T, Loenneke JP. Influence of cuff material on blood flow restriction stimulus in the upper body. J Physiol Sci 2017; 67: 207-215

[8] Counts BR, Dankel S], Barnett BE, Kim D, Mouser JG, Allen KM, Thiebaud RS, Abe T, Bemben MG, Loenneke JP. Influence of relative blood flow restriction pressure on muscle activation and muscle adaptation. Muscle Nerve 2016; 53: 438-445

[9] Dankel S], Jessee MB, Abe T, Loenneke JP. The effects of blood flow restriction on upper-body musculature located distal and proximal to applied pressure. Sports Med 2016; 46: 23-33

[10] Harriss D], Atkinson G. Ethical standards in sport and exercise science research: 2016 update. Int J Sports Med 2015; 36: 1121-1124

[11] Jessee MB, Buckner SL, Dankel SJ, Counts BR, Abe T, Loenneke JP. The influence of cuff width, sex, and race on arterial occlusion: Implications for blood flow restriction research. Sports Med 2016; 46: 913-921

[12] Jessee MB, Buckner SL, Mouser JG, Mattocks KT, Loenneke JP. Letter to the editor: Applying the blood flow restriction pressure: The elephant in the room. Am J Physiol 2016; 310: H132-H133

[13] Kacin A, Strazar K. Frequent low-load ischemic resistance exercise to failure enhances muscle oxygen delivery and endurance capacity. Scand J Med Sci Sports 2011; 21: e231-e241

[14] Laurentino GC, Loenneke JP, Teixeira EL, Nakajima E, lared W, Tricoli V. The effect of cuff width on muscle adaptations after blood flow restriction training. Med Sci Sports Exerc 2016; 48: 920-925

[15] Laurentino GC, Ugrinowitsch C, Roschel H, Aoki MS, Soares AG, Neves M Jr., Aihara AY, Fernandes Ada R, Tricoli V. Strength training with blood flow restriction diminishes myostatin gene expression. Med Sci Sports Exerc 2012; 44: 406-412
[16] Lixandrão ME, Ugrinowitsch C, Laurentino G, Libardi CA, Aihara AY, Cardoso FN, Tricoli V, Roschel H. Effects of exercise intensity and occlusion pressure after 12 weeks of resistance training with blood-flow restriction. Eur J Appl Physiol 2015; 115: 2471-2480

[17] Loenneke JP, Allen KM, Mouser JG, Thiebaud RS, Kim D, Abe T, Bemben MG. Blood flow restriction in the upper and lower limbs is predicted by limb circumference and systolic blood pressure. Eur ] Appl Physiol 2015; 115: 397-405

[18] Loenneke JP, Fahs CA, Rossow LM, Sherk VD, Thiebaud RS, Abe T, Bemben DA, Bemben MG. Effects of cuff width on arterial occlusion: Implications for blood flow restricted exercise. Eur J Appl Physiol 2012; 112: 2903-2912

[19] Loenneke JP, Kim D, Fahs CA, Thiebaud RS, Abe T, Larson RD, Bemben DA, Bemben MG. The effects of resistance exercise with and without different degrees of blood-flow restriction on perceptual responses. J Sports Sci 2015; 33: 1472-1479

[20] Loenneke JP, Kim D, Mouser JG, Allen KM, Thiebaud RS, Abe T, Bemben MG. Are there perceptual differences to varying levels of blood flow restriction? Physiol Behav 2016; 157: 277-280

[21] Loenneke JP, Thiebaud RS, Abe T, Bemben MG. Blood flow restriction pressure recommendations: the hormesis hypothesis. Med Hypotheses 2014; 82: 623-626

[22] Motykie GD, Zebala LP, Caprini JA, Lee CE, Arcelus JI, Reyna J, Cohen EB. A guide to venous thromboembolism risk factor assessment. J Thromb Thrombolysis 2000; 9: 253-262

[23] Rossow LM, Fahs CA, Loenneke JP, Thiebaud RS, Sherk VD, Abe T, Bemben MG. Cardiovascular and perceptual responses to blood-flowrestricted resistance exercise with differing restrictive cuffs. Clin Physiol Funct Imaging 2012; 32: 331-337

[24] Spranger MD, Krishnan AC, Levy PD, O'Leary DS, Smith SA. Blood flow restriction training and the exercise pressor reflex: A call for concern. Am J Physiol 2015; 309: H1440-H1452

[25] Takarada Y, Nakamura Y, Aruga S, Onda T, Miyazaki S, Ishii N. Rapid increase in plasma growth hormone after low-intensity resistance exercise with vascular occlusion. J Appl Physiol 2000; 88: 61-65

[26] Takarada Y, Nozaki D, Taira M. Force overestimation during tourniquetinduced transient occlusion of the brachial artery and possible underlying neural mechanisms. Neurosci Res 2006; 54: 38-42

[27] Teixeira EL, Barroso R, Silva-Batista C, Laurentino GC, Loenneke JP, Roschel H, Ugrinowitsch C, Tricoli V. Blood flow restriction increases metabolic stress but decreases muscle activation during high-load resistance exercise. Muscle Nerve 2017, doi:10.1002/mus.25616

[28] Yasuda T, Abe T, Brechue WF, lida H, Takano H, Meguro K, Kurano M, Fujita S, Nakajima T. Venous blood gas and metabolite response to low-intensity muscle contractions with external limb compression. Metabolism 2010; 59: 1510-1519

[29] Yasuda T, Brechue WF, Fujita T, Sato Y, Abe T. Muscle activation during low-intensity muscle contractions with varying levels of external limb compression. J Sports Sci Med 2008; 7: 467-474 